\title{
System Performance and Limits of Optical Modulation Formats in Dense Wavelength Division Multiplexing Systems
}

\author{
Rajdi Agalliu ${ }^{1}$, Michal Lucki ${ }^{1}$ \\ ${ }^{I}$ Department of Telecommunication Engineering, Faculty of Electrical Engineering, Czech Technical \\ University in Prague, \\ Technicka 2, 16627 Prague 6, Czech Republic \\ agallraj@fel.cvut.cz
}

\begin{abstract}
In this paper we investigate in OptSim software environment the system performance of intensity and phase modulation formats for different network scenarios and dense wavelength division multiplexing grids. OptSim employs the Time Domain Split Step method to implement the signal distribution equation in a fiber. We investigate intensity formats, such as Non Return to Zero, Return to Zero, CarrierSuppressed Return to Zero and DuoBinary, and phase modulation formats like Differential Phase-Shift Keying and Differential Quadrature Phase-Shift Keying. The main goal is to compare these formats in terms of bit error rate, $Q$-factor, optical reach and grid limitations for transmission rates 10,40 and 100 Gbps per channel and discuss the possibilities of increasing their spectral efficiency. We also focus on other advanced solutions such as the polarization division multiplexing combined with phase modulations, coherent detection and advanced digital signal processing which mainly benefits in spectral efficiency, optical signal to noise ratio and chromatic dispersion tolerances.
\end{abstract}

Index Terms-Modulation, optical fiber communication, optical transmitters, WDM networks.

\section{INTRODUCTION}

The increasing demand for higher transmission rates over the existing fiber infrastructure is creating the necessity on finding other advanced and cost effective solutions and one of them could be the modeling of new modulation formats capable of speeds over $100 \mathrm{Gbps}$ per channel. This requires detailed knowledge on shortcomings and limits of currently implemented modulations which usually enable in dense wavelength division multiplexing systems transmission rates of $10 \mathrm{Gbps}, 40 \mathrm{Gbps}$ and $100 \mathrm{Gbps}$ per channel. Some formats cannot offer higher rates due to their hardware limitations. Issues like Polarization Mode Dispersion and nonlinear effects have to be solved to achieve lower Bit Error Rates (BER) at the receiver. We use OptSim [1], [2] to investigate the performance of the intensity, phase and other advanced modulation formats for different dense wavelength division multiplexing (DWDM) grids and transmission rates

Manuscript received 26 April, 2015; accepted 28 January, 2016.

This work has been supported by the CTU grand under project SGS16/227/OHK3/3T/13. of $10 \mathrm{Gbps}, 40 \mathrm{Gbps}$ and $100 \mathrm{Gbps}$ per channel for a given network topology. Investigated formats include Non Return to Zero (NRZ), Return to Zero (RZ), Carrier-Suppressed Return to Zero (CSRZ), DuoBinary (DB), Differential Phase-Shift Keying (DPSK), Differential Quadrature PhaseShift Keying (DQPSK) and Polarization Division Multiplexing Quadrature Phase-Shift Keying (PDM-QPSK).

\section{STATE OF ART}

Multilevel intensity modulation formats deal with significant back to back receiver sensitivity penalties, therefore the attention in this paper is paid primarily to the binary intensity formats. In reference [3], authors extensively compare intensity and phase modulation formats with respect to chromatic dispersion, polarization-mode dispersion, WDM crosstalk, optical amplifier noise, narrowband optical filtering and fibre nonlinearity. In this paper we investigate the most two common intensity formats, i.e. NRZ and RZ, as well as CSRZ. DB is another format considered in the simulation setups, due to its high tolerance to chromatic dispersion and narrowband optical filtering. On the other hand, phase modulation formats offer higher spectral efficiency and optical signal-to-noise ratio (OSNR) tolerance at the cost of transceiver's complexity.

In [4], authors investigate different types of formats, such as NRZ, RZ, CRZ, CSRZ, DB, RZ-DPSK, NRZ-DPSK applied in WDM-PONs for link distances from up to $50 \mathrm{~km}$ and rates $1.25 \mathrm{Gbps}, 2.5 \mathrm{Gbps}$ and $10 \mathrm{Gbps}$. The study given in [5] shows a detailed performance comparison of various types of DB and phase shaped binary transmission (PSBT) formats for $40 \mathrm{Gbps}$ and mixed 10/40 Gbps long-haul WDM transmission systems based on standard single mode fiber (SSMF) and LEAF. Both DB and PSBT formats are more robust to intra-channel Kerr nonlinear effects than NRZ. DB generally offers an acceptable compromise between robustness to OSNR degradation and chromatic dispersion immunity. On the other hand, DB is a little bit less resistant to differential group delay than NRZ. DPQSK is primarily limited by cross-phase modulation (XPM) and is more suitable for transmission based on SSMF.

Authors in [6] evaluate the performance of 42.7 Gbps 
DWDM systems for formats such as NRZ-OOK, DB, NRZDBPSK and RZ-DQPSK. Results from simulations show that RZ-DQPSK combined with LEAF for $50 \mathrm{GHz}$ channel spacing and spectral efficiency of $0.8 \mathrm{bit} / \mathrm{s} / \mathrm{Hz}$ can provide approximately $50 \%$ improvement in terms of transmission distance over implementations based on other fiber and modulation formats. In [7], DPSK and DQPSK formats with NRZ, $33 \%$ RZ and CSRZ are investigated in 160 Gbps channels. Chromatic dispersion, higher-order chromatic dispersion, nonlinearity and OSNR are considered as well. Simulation results show that RZ-DQPSK offers the longest reach, NRZ-DQPSK offers the highest dispersion tolerance and RZ-DPSK enables the highest nonlinearity tolerance.

Other advanced solutions have recently been designed such as PDM-QPSK. PDM has been widely denoted either by polarization multiplexing, polarization division multiplexing, dual polarization or orthogonal polarization [8]. PDM-QPSK combined with coherent detection [9] and advanced digital signal processing offers a very promising modulation format designed primarily for $100 \mathrm{Gbps}$ optical channels. Authors in reference [10], investigate the performance of $100 \mathrm{Gbps}$ PDM-QPSK channels for 8 Tbps transmission over a dispersion managed link based on low dispersion fibers. Experiment in reference [11] compares the system performance of $80 \mathrm{Gbps} \times 112 \mathrm{Gbps}$ long-haul PDM-QPSK DWDM transmission over large-area fiber and SSMF spans. Other modulation formats for $100 \mathrm{Gbps}$ and higher rates are recently under research, e.g. the Dual Polarization Multi-Band OFDM (DP-MB-OFDM) format. Authors in reference [12] show that DP-MB-OFDM and PDM-QPSK offer nearly the same performance at $100 \mathrm{Gbps}$ after transmission over a $10 \mathrm{~km} \times 100 \mathrm{~km}$ fiber line. Another promising modulation format for transmission rates 100 Gbps and especially for those higher [13], [14] is the combination of polarization division multiplexing with Quadrature Amplitude Modulation (QAM) formats. Experiment in reference [15] shows the suitability of 256 Gbps PM-16QAM and 128 Gbps PM-QPSK modulated signals in long-haul and submarine systems with span lengths over $100 \mathrm{~km}$.

\section{METHODS}

The results are obtained using OptSim, which employs the Time Domain Split Step (TDSS) method to realize the signal propagation through the fiber. The monitors we use are eye diagrams, BER and Q-factor. In this section we also describe the simulation setups, their components and parameters.

\section{A. Time Domain Split Step method}

The propagation equation that the TDSS method solves is

$$
\frac{\partial A(t, z)}{\partial z}=\{L+N\} \times A(t, z),
$$

where $A(t, z)$ describes the complex envelope, $L$ linear effects, and $N$ non-linear effects [2]. The Split-Step algorithm applies $L$ and $N$ components separately to calculate $A(t, z)$ over small fibre spans. OptSim uses TDSS method to calculate the operator for linear effects in the time domain by applying convolution in sampled time [2]

$$
A_{L}[n]=A[n] \times h[n]=\sum_{k=-\infty}^{\infty} A[k] \times h[n-k],
$$

where $h$ is the impulse response of $L$.

\section{B. Bit Error Rate, Eye Diagram and Q-factor}

The monitors we mainly use in this paper are BER, eye diagrams, and Q-factor. From the eye diagram's graphical appearance we could judge the amount of noise, intersymbol interference, jitter and signal distortion. Q-factor specifies the minimum required OSNR to get a certain BER. It can be expressed from the mean values $\mu$ of logical 0 and 1 and their corresponding standard deviations $\sigma[16]$

$$
Q[-]=\frac{\mu_{1}-\mu_{0}}{\sigma_{1}+\sigma_{0}} .
$$

Q-factor and BER are related as follows [16]

$$
\operatorname{BER}[-]=\frac{1}{2} \times \operatorname{erfc}\left(\frac{Q}{\sqrt{2}}\right) .
$$

\section{Transceivers}

In this section we describe the transceiver's design for each of the investigated formats [17], [18]. The NRZ transmitter is based on a continuous wave laser externally modulated with a Mach-Zehnder modulator (MZM). We set parameters, such as bit rate, laser frequency, full width at half maximum, output power of the laser, loss inserted by the modulator, etc. The RZ transmitter's design is similar to that of NRZ. Unlike NRZ, we set the RZ raised cosine format in the electrical signal generator. For the CSRZ type, a NRZ optical generated signal is subsequently modulated by a second MZM, which is driven by a sinusoidal signal with a frequency half of the bit rate. Any two adjacent bits will have a $\pi$ phase shift and it is possible to suppress the carrier and generate a CSRZ modulated signal.

The DB transmitter consists of an amplitude dual-arm MZM with two electrical inputs: the first one is the standard electrical signal achieved by pseudorandom binary sequence signals passing through a NRZ driver a low-pass filter, and the second input by performing a bitwise logical NOT operation on the logical input which again travels through a driver and an electrical filter. Similarly as for NRZ, a continuous wave laser is used as a light source. A similar receiver as in the previous modulation formats is used.

NRZ-DPSK transmitter's design is similar to that of NRZ, but instead of the MZM, a phase modulator with $180^{\circ}$ phase shift is deployed. A low-pass filter is used between the driver and modulator to include the impact of a non-ideal binary to electrical signal conversion. The RZ-DPSK transmitter includes additionally among others a second modulator to generate RZ output pulses.

In a NRZ-DQPSK transmitter, the two input encoded inphase and quadrature binary signals are converted into electrical waveforms which drive two MZMs. A continuous laser source is used for both MZMs. The output of one of the modulators travels through a phase modulator used to obtain an additional phase shift of $90^{\circ}$, which is required for the quadrature component. Signals are then combined together to generate a single DQPSK modulated signal. Additional 
components include two low pass filters between electrical signal generators and MZMs to consider also the non-ideal binary to electrical signal conversion. For RZ-DQPSK, an additional MZM is used to create the RZ output pulses.

In a PDM-QPSK transmitter we have to deal with four signal components for the in-phase respectively quadrature signals and for the two polarizations. Similarly as in previous formats, the binary to electrical signal conversion is performed, and low pass Bessel filters are used again to consider the impact of a non-ideal conversion. The four electrical signals are then launched to two QPSK modulators, two per each. The output signal of one of the modulators travels through a polarization rotator and then it is combined with the output from the second QPSK modulator to obtain a single PDM-QPSK modulated signal.

For NRZ, RZ and CSRZ, we use a compound receiver which models a photodetector, an electrical amplifier and an electrical filter. A DPSK receiver consists of a delay interferometer for decoding purposes and a balanced receiver modeled using two regular compound receivers, where the electrical output of the first one is added to the second's inverted output. Meanwhile a DQPSK receiver is modeled using two balanced 2DPSK receivers for the inphase and quadrature signals, each composed of a tunable Mach Zehnder interferometer and two PIN photodetectors. The PDM-QPSK receiver includes many components such as a single ended $90^{\circ}$ hybrid with local oscillator and four PIN photodiodes to enable the coherent detection; transimpedance amplifiers, electrical filters, electronic dispersion compensator and in the end a memoryless blind receiver to separate the in-phase and quadrature components and orthogonal polarizations.

\section{Simulation Setups}

The overall system performance of certain modulations is degraded beyond a certain transmission rate (results in section IV) and their deployment in a DWDM network starts to become inefficient without further transceiver's improvement. Such formats have been excluded from the comparison after a certain bit rate in order to figure out the most appropriate format in the assumed network scenario for a given transmission rate. We investigate two passive optical network topologies. The first one is based on a single fiber to primarily find the maximum reach (Fig. 1).

The second one (Fig. 2) is a tree topology to find out how formats could run in a typical optical access network.

Several transmitters are used to generate the DWDM channels with approx. $1550 \mathrm{~nm}$ central channel. Transmitter's optical power is set to $0 \mathrm{dBm}$ for comparison purposes. We use SSMF with $0.2 \mathrm{~dB} / \mathrm{km}$ of loss. WDM multiplexers and demultiplexers are modelled as a set of filters whose parameters have been optimized for each modulation format. The goal is to achieve as higher spectral efficiency as possible. The measurement components we use include signal analysers, spectrum analysers, BER testers, eye diagram analysers, optical power meters, etc.

PDM-QPSK is primarily designed for $100 \mathrm{Gbps}$ channel systems and our purpose of simulating this format in OptSim is to figure out its suitability in long haul transmission systems. For this reason, another type of the simulation design has been used. The optical signal is noise loaded to extract the receiver BER as the function of OSNR [19] as schematically shown in Fig. 3. We use non-zero dispersion shifted fibers with $0.2 \mathrm{~dB} / \mathrm{km}$ of loss and chromatic dispersion around $4 \mathrm{ps} / \mathrm{nm} / \mathrm{km}$ at the considered band. Each fiber span length is set to $100 \mathrm{~km}$ and separated from each other by optical amplifiers with a fixed gain equal to $20 \mathrm{~dB}$.

We also consider in the PDM-QPSK transmitter a $7 \%$ bit rate overhead for forward error correction. The simulation results are given in Section IV part C.

\section{RESUltS AND DisCUSSION}

\section{A. Intensity Modulation Formats}

NRZ has been widely deployed due to its easy generation, cost efficiency and sufficient bandwidth to fulfill the network requirements.

Fig. 1. Standard topology for 10, 40 and 100 Gbps DWDM systems.
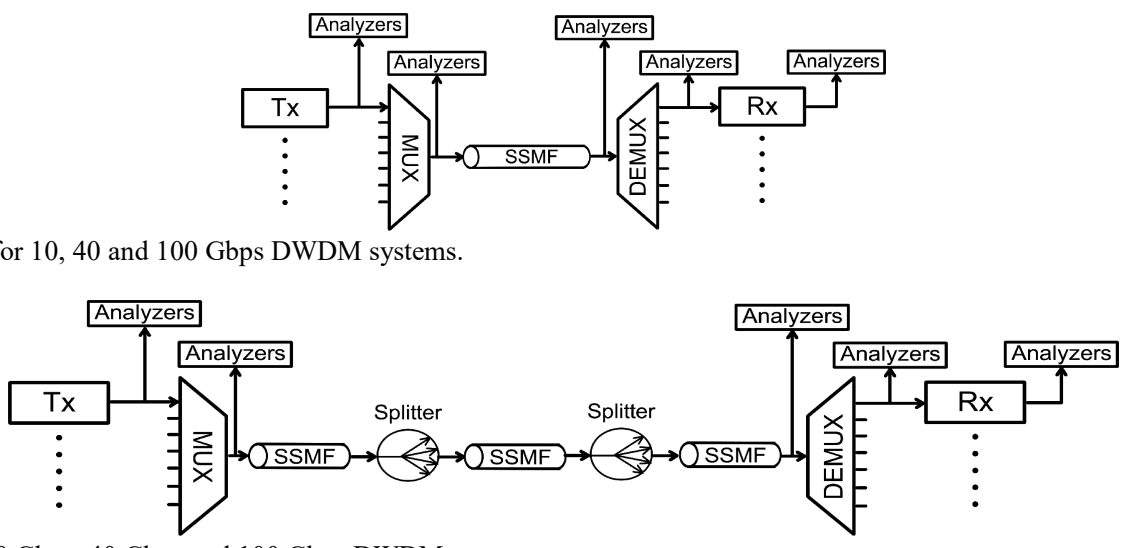

Fig. 2. Tree topology for $10 \mathrm{Gbps}, 40 \mathrm{Gbps}$ and $100 \mathrm{Gbps}$ DWDM systems.

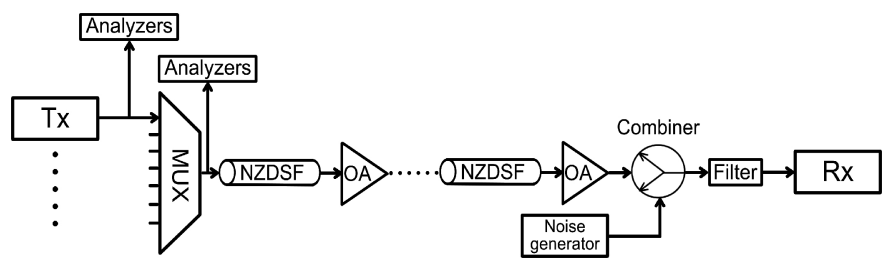

Fig. 3. Simulation scheme for the 100 Gbps PDM-QPSK DWDM system. 
In this section we discuss this format and compare it with other intensity formats. In Fig. 4, we show optical spectra of intensity formats that we have investigated for $10 \mathrm{Gbps}$ and 40 Gbps transmission systems. Each transmitter's output power is set to $0 \mathrm{dBm}$ and the operating wavelength to 1550 nm. A spectrum analyser is placed directly after the transmitter for simplification purposes. The scale of the $\mathrm{x}$ axis has been adjusted for each transmission rate for comparison purposes. The NRZ format has a narrower main lobe than other formats. One can observe the spectrum broadening of RZ while comparing it with NRZ, which results in an ineffective bandwidth utilization. However $\mathrm{NRZ}$ is prone to chromatic dispersion and nonlinear effects like XPM due to the fact that bits occupy the entire bit slots and this has a strong impact on NRZ's limitations. As mentioned in section III part C, the CSRZ modulated signal introduces a $\pi$ phase shift between adjacent bits resulting in carrier suppression (Fig. 4) which can reduce the interference between adjacent pulses. In the first step, each of the three formats was simulated according to the topology in Fig. 1. Results showed that the acceptable DWDM grid starts from $25 \mathrm{GHz}$. NRZ and CSRZ could achieve at least $10^{-10}$ BER up to $80 \mathrm{~km}$ of reach with the proper use of optical filters in the $10 \mathrm{Gbps}$ DWDM system with $25 \mathrm{GHz}$ channel spacing. RZ did not perform well primarily due to its broader spectrum and higher interference between adjacent channels.

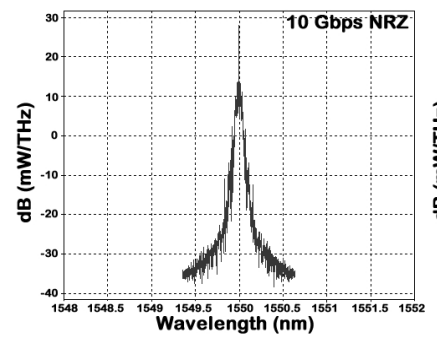

(a)

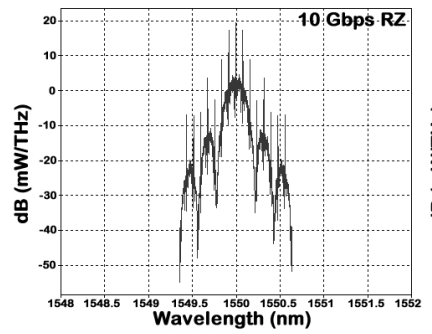

(c)

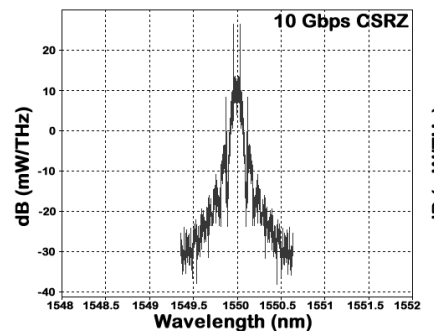

(e)

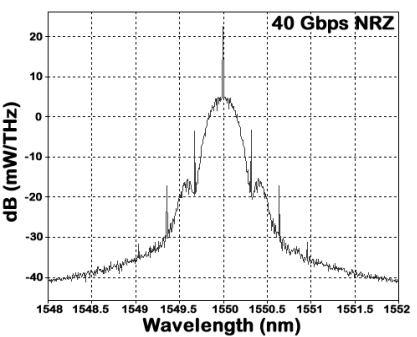

(b)

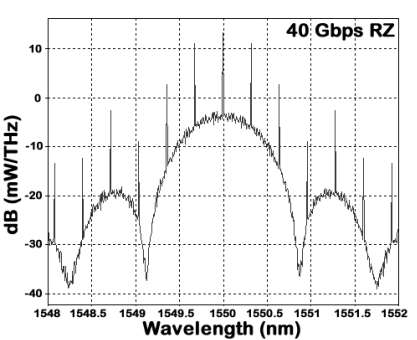

(d)

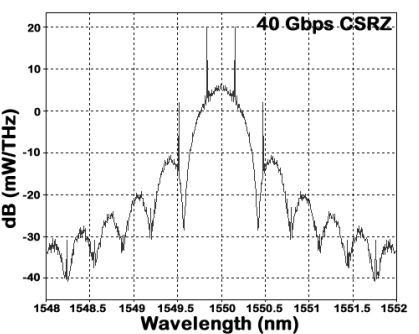

(f)
Fig. 4. Optical spectra of intensity modulation formats for $10 \mathrm{Gbps}$ and 40 Gbps.

Similar results were also achieved for the tree topology. As an example, NRZ and CSRZ could enable up to $24 \mathrm{~km}$ of reach consisting of three fiber spans: $12 \mathrm{~km}, 8 \mathrm{~km}$ and $4 \mathrm{~km}$ separated by 1:8 and 1:32 splitters (Fig. 2). Nevertheless, there was seen a slight improvement in terms of BER by CSRZ due to its suppressed carrier which reduces interchannel interferences. The transition to higher transmission rates emphasis the shortcomings of every format as can be expected for the spectra shown in Fig. 4. As for 40 Gbps and higher, all formats did not perform well due to their significant increased crosstalk penalties, resulting in a highly deteriorated performance, especially in DWDM systems.

Subsequently, we investigate the DB format. The results from spectrum analysers are shown in Fig. 5 for a DB modulated signal at $10 \mathrm{Gbps}$ and $40 \mathrm{Gbps}$.

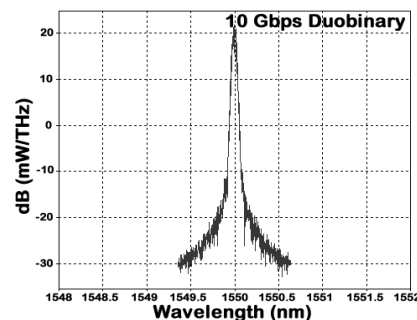

(a)

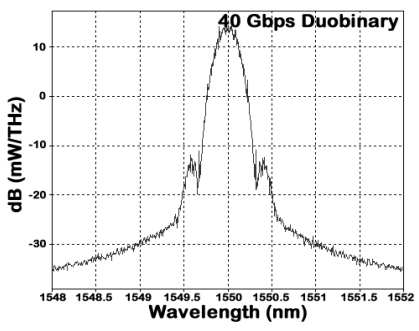

(b)
Fig. 5. Optical spectra of DB for $10 \mathrm{Gbps}$ (a) and $40 \mathrm{Gbps}$ (b)

As can be seen, its spectrum gets much broader, as the transmission rate increases. The DB format primarily benefits in high chromatic dispersion tolerance and narrowband optical filtering. This could potentially be applicable for increasing the spectral efficiency in WDM systems. The minimum channel spacing was found to be 12.5 $\mathrm{GHz}$ for $10 \mathrm{Gbps}$ transmission systems. In such scenario, DB is capable of offering up to $110 \mathrm{~km}$ of optical reach, which clearly proves the suitability of this format at this speed over the previous intensity format. Deployment of $25 \mathrm{GHz}$ channel spacing could offer up to $27 \%$ improvement in terms of the maximum optical reach. For this scenario, the DB format was also simulated for the tree topology. The minimum BER was obtained at these SSMF lengths: $25 \mathrm{~km}, 15 \mathrm{~km}, 5 \mathrm{~km}$, separated by $1: 8$ and $1: 32$ splitters similarly as for the previous formats. DB's immunity to nonlinear effects especially at higher transmission rates is not that different from previous binary modulation formats. DB could only offer up to $15 \mathrm{~km}$ of optical reach in a 40 Gbps DWDM system with $100 \mathrm{GHz}$ channel spacing. In order to achieve better results, the dispersion map optimization is necessary among others.

\section{B. Phase Modulation Formats}

In this section we discuss the results for phase based formats such as DPSK, RZ-DSPK, DQPSK and RZDQPSK. In Fig. 6, we show optical spectra of DPSK and RZ-DPSK for $10 \mathrm{Gbps}$ and $40 \mathrm{Gbps}$.

The design of transmitters was described in Section III part C. For comparison purposes we have adjusted the scale of the $\mathrm{x}$ axis to show how spectra differ from each other. As can be seen from Fig. 6, DPSK's spectrum at 40 Gbps starts to be much wider, which extensively decreases its efficiency especially for DWDM systems at such transmission rate and becomes deprecated for higher ones. Simulations showed that phase-based formats offer higher spectral efficiency 
while comparing with OOKs at the cost of transceiver's complexity.

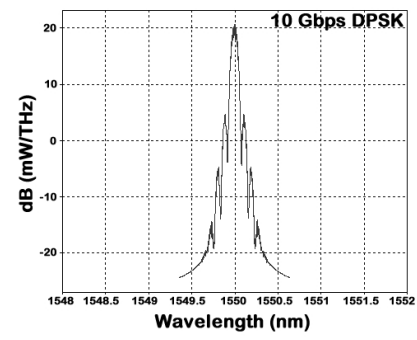

(a)

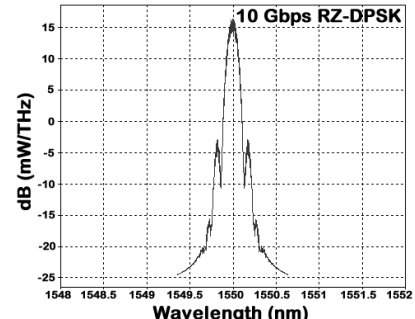

(c)

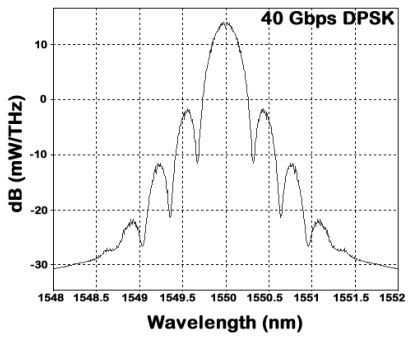

(b)

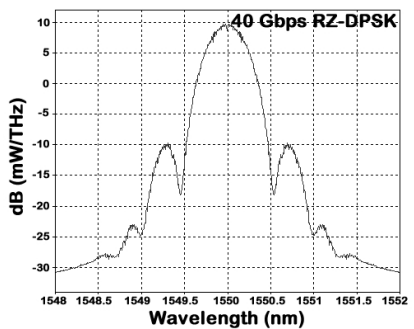

(d)
Fig. 6. Optical spectra of DPSK and RZ-DPSK for $10 \mathrm{Gbps}$ and $40 \mathrm{Gbps}$.

At first glance, DPSK's narrow main lobe could offer higher spectral efficiency than RZ-DPSK. On the other side RZ-DPSK could achieve higher crosstalk penalties. For 100 Gbps, DPSK's performance significantly degrades. We compare both formats in DWDM systems (Fig. 1) in terms of BER, Q-factor and maximum optical reach. The minimum acceptable channel spacing at $10 \mathrm{Gbps}$ was found to be $25 \mathrm{GHz}$. In such scenario, DPSK can offer up to $105 \mathrm{~km}$ of optical reach. On the other hand RZ-DPSK offers $6 \%$ less, primarily due to its wider spectrum (Fig. 6), which causes at this grid higher interference between adjacent channels. Both formats were also simulated for the tree topology shown in Fig. 2. DPSK can offer up to $100 \mathrm{~km}$ reach consisting of $60 \mathrm{~km}, 30 \mathrm{~km}$, and $10 \mathrm{~km}$ fiber spans, which are separated by splitters 1:8 and 1:32. RZ-DPSK's result is very similar, and there is only a slightly better result achieved by DPSK, which offers approx. $5 \mathrm{~km}$ longer reach.

DPSK and RZ-DPSK are also compared at 40 Gbps. Their grid limitation was found to be $100 \mathrm{GHz}$ for the assumed topologies. The system performance of both formats was significantly decreased. For such a reason, SSMFs used in these simulations were replaced by the world's most widely deployed Non-Zero Dispersion-Shifted Fiber, i.e. Corning LEAF. As a result, DPSK could enable up to $26 \mathrm{~km}$ optical reach and RZ-DPSK around $8 \%$ more, for a minimum BER of $10^{-10}$. Implementation of these formats in the tree topology with SSMFs shown in Fig. 2 is not efficient. Both formats were not able to reach over $10 \mathrm{~km}$ due to the high insertion loss of 1:8 and 1:32 splitters. Other splitters with a lower split ratio have to be used and LEAFs can be deployed again instead of SSMFs.

In the following part, we investigate DPQSK and RZDQPSK. In Fig. 7, we show their optical spectra that we obtained from spectrum analysers in OptSim.

DPQSK's compression in frequency offers higher spectral efficiency compared to DPSK primarily at $10 \mathrm{Gbps}$, as well as increased tolerance to chromatic dispersion and polarization mode dispersion due to its longer symbol length. DQPSK can double the bit rate for a given bandwidth, or it can halve the required bandwidth for a certain bit rate. The numerical results showed that DQPSK could achieve after optimizing the simulation parameters a maximum reach of $100 \mathrm{~km}$ for at least $10^{-10} \mathrm{BER}$, without using any active component in the optical path in a $10 \mathrm{Gbps}$ transmission system with $12.5 \mathrm{GHz}$ channel spacing (Fig. 1). Larger channel spacing significantly decreases the interchannel interference. In case of DQPSK, a channel spacing of $25 \mathrm{GHz}$ could achieve up to $40 \%$ reach improvement. RZ-DQPSK improves the system performance at the cost of the transmitter's complexity. This format could offer up to $130 \mathrm{~km}$ of reach for $12.5 \mathrm{GHz}$ channel spacing and $45 \%$ more for $25 \mathrm{GHz}$ spacing. The tree topology network has been tested as well. The best result was achieved by $10 \mathrm{Gbps}$ RZ-DQPSK system with $25 \mathrm{GHz}$ spacing, which could offer up to $180 \mathrm{~km}$ reach consisting of $110 \mathrm{~km}, 60 \mathrm{~km}$ and $10 \mathrm{~km}$ fiber spans (Fig. 2), separated by 1:8 and 1:32 splitters.

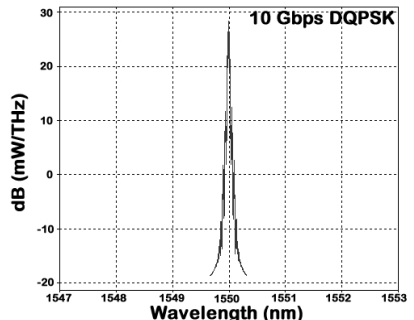

(a)

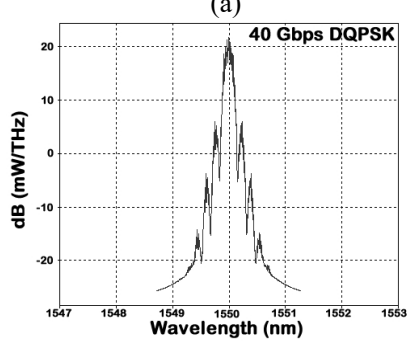

(c)

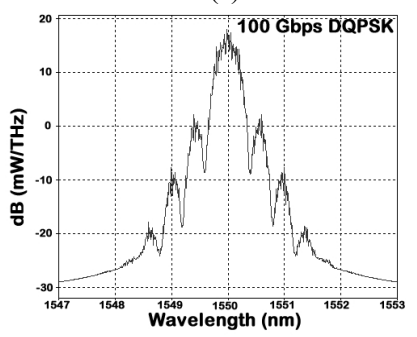

(e)

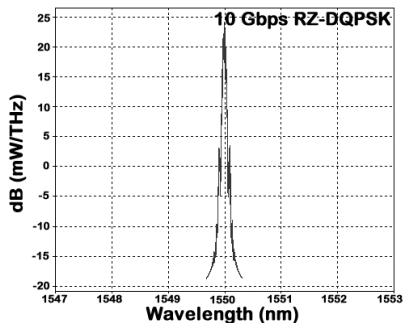

(b)

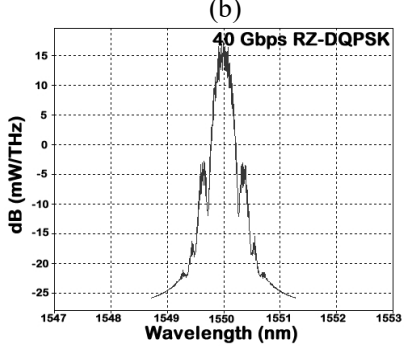

(d)

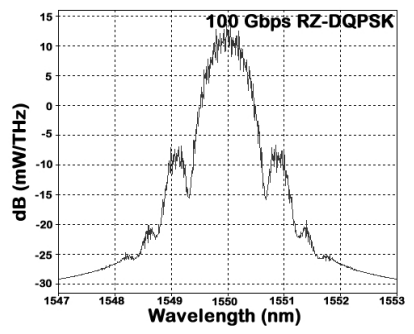

(f)
Fig. 7. Spectra of DQPSK and RZ-DQPSK for $10 \mathrm{Gbps}, 40 \mathrm{Gbps}$ and 100 Gbps.

Subsequently, 40 Gbps DQPSK modulated signals were investigated. The system performance using SSMFs was extensively decreased. Similarly as for DPSK, SSMF was replaced by LEAF. Simulations showed that 40 Gbps DQPSK systems with a single channel and LEAF could reach up to $45 \mathrm{~km}$ and RZ-DQPSK about $7 \%$ more. Based on this setup, we built in OptSim several 40 Gbps DQPSK DWDM scenarios. The limitation was found to be at $50 \mathrm{GHz}$ spacing, for which the system could offer up to $35 \mathrm{~km}$ of reach to obtain at least $10^{-10} \mathrm{BER}$. Results showed also that RZ-DQPSK's maximum reach is in this case $10 \%$ lower for the same simulation parameters, primarily due to its wider main lobe which causes a higher inter-channel interference. 
As can be expected from Fig. 7, simulations showed that the performance of $100 \mathrm{Gbps}$ DQPSK DWDM system based on LEAF is not efficient without further improvements in transceivers, deployment of optical amplifiers, etc. The maximum reach did not cross $10 \mathrm{~km}$ and the maximum allowed spacing was found to be $100 \mathrm{GHz}$. Next session will show that PDM-QPSK proves to be the appropriate modulation at $100 \mathrm{Gbps}$.

\section{Polarization Division Multiplexing}

In this section we discuss the results related to PDMQPSK. This format has been simulated only for $100 \mathrm{Gbps}$ since other formats without such a transceiver as PDMQPSK's one (Section III part C) perform well at lower transmission rates and accomplish the contemporary infrastructure needs. Its optical spectrum is shown in Fig. 8.

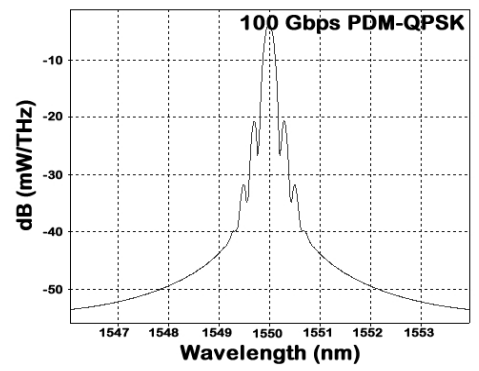

Fig. 8. Optical spectrum of a 100 Gbps PDM-QPSK modulated signal.

As it can be seen from the spectrum, this format can offer high spectral efficiency although it deals with transmission rate 100 Gbps per channel. The PDM-QPSK coherent detection provides high sensitivity receivers making it a very promising format for standardization. PDM increases the transmission capacity while comparing with DQPSK or other multilevel formats. The simulation results that we obtained in OptSim are based on the scheme shown in Fig. 3. We could find that the maximum allowed channel spacing is $50 \mathrm{GHz}$. By properly setting the parameters on transceivers, including those of optical filters, PDM-QPSK could enable $2000 \mathrm{~km}$ of optical reach, i.e. 20 fiber spans of $100 \mathrm{~km}$ each, separated by inline amplifiers with fixed gains $20 \mathrm{~dB}$ (Fig. 3). For this reach, a total pre-FEC value for all four signals was found to be in the order of $10^{-4}$ for $20 \mathrm{~dB}$ span loss, which demonstrates the capability of this format of performing well even after such a long transmission system without any inline dispersion compensation.

\section{CONCLUSIONS}

In this paper we investigated intensity, phase and PDM based modulation formats for optical communications. The main goal was to find out their limitations in commonly used network topologies. RZ was found to be mainly limited by its broader spectrum and higher inter-channel interference compared to other investigated intensity formats. CSRZ improves the overall system performance in terms of BER and maximum optical reach due to its suppressed carrier. However, CSRZ and NRZ offered similar results in $10 \mathrm{Gbps}$ DWDM networks with the minimum acceptable channel spacing of $25 \mathrm{GHz}$. Transition to higher bit rates significantly decreased the performance of all these intensity formats. On the other hand, DB proved its suitability over the other intensity formats due to its high chromatic dispersion tolerance and narrow spectral width. DB could enable over $100 \mathrm{~km}$ of optical reach for a $10 \mathrm{Gbps}$ DWDM system with $12.5 \mathrm{GHz}$ channel spacing. Although at $40 \mathrm{Gbps}$ DB was more efficient than other formats, its immunity to nonlinear effects was not that different. Phase-based modulations offered high spectral efficiency at the cost of transceiver's complexity. As an example, both DPSK and RZ-DPSK could enable around $100 \mathrm{~km}$ of reach for a 10 Gbps DWDM system with $25 \mathrm{GHz}$ spacing. At $40 \mathrm{Gbps}$ results were quite poor, and improvement was achieved by replacing SSMFs with LEAFs. DQPSK and RZ-DQPSK offered higher spectral efficiencies due to their compression in frequency, increased tolerance to chromatic dispersion as well as to polarization mode dispersion, compared to DPSK and RZ-DPSK. DQPSK could enable up to $100 \mathrm{~km}$ of reach in a $10 \mathrm{Gbps}$ system with $12.5 \mathrm{GHz}$ spacing and RZ-DQPSK around $30 \%$ more. Significant improvements were observed for both formats while choosing larger channel spacing. At 40 Gbps, both formats performed well while deploying LEAFs instead of SSMFs. The maximum optical reach was $35 \mathrm{~km}$ for the lowest acceptable channel spacing, i.e. 50 $\mathrm{GHz}$. At $100 \mathrm{Gbps}$ the only efficient modulation was found to be PDM-QSPK, which combines the benefits of phasebased modulations with PDM, as well as coherent detection and advanced signal processing, at the cost of transceiver's complexity. 100 Gbps PDM-QPSK system was successfully simulated for a $2000 \mathrm{~km}$ DWDM system with $50 \mathrm{GHz}$ channel spacing and without inline dispersion compensation.

\section{REFERENCES}

[1] OptSim Application Notes and Examples, RSOFT Design Group, Inc., 2010.

[2] OptSim User Guide, RSOFT Design Group, Inc., 2010

[3] P. J. Winzer, R. J. Essiambre, "Advanced modulation formats for high-capacity optical transport networks", Journal of Lightwave Technology, vol. 24, no. 12, pp. 4711-4728, 2006. [Online]. Available: http://dx.doi.org/10.1109/JLT.2006.885260

[4] J. Latal, J. Vitasek, P. Koudelka, P. Siska, R. Poboril, L. Hajek, A. Vanderka, V. Vasinek, "Simulation of modulation formats for optical access network based on WDM-PON", in 16th Int. Conf. Transparent Optical Networks (ICTON), 2014. [Online]. Available: http://dx.doi.org/10.1109/icton.2014.6876473

[5] A. Tan, E. Pincemin, "Performance comparison of duobinary formats for $40-\mathrm{Gb} / \mathrm{s}$ and mixed 10/40-Gb/s long-haul WDM transmission on SSMF and LEAF Fibers", in Journal of Lightwave Technology. vol. 27, no. 4, 2009. [Online]. Available: http://dx.doi.org/10.1109/ JLT.2008.929117

[6] S. Ghoniemy, K. F. George, L. MacEachern, "Performance evaluation and enhancements of $42.7 \mathrm{~Gb} / \mathrm{s}$ DWDM transmission system using different modulation formats", in Ninth Annual Communication Networks and Services Research Conf., 2011, pp. 189-194. [Online]. Available: http://dx.doi.org/10.1109/CNSR.2011.35

[7] D. Wang, D. Lu, C. Lou, L. Huo, W. Yu, "Performance comparison of phase modulated formats in $160 \mathrm{~Gb} / \mathrm{s}$ transmission system", in Communications and Photonics Conf. and Exhibition, ACP, Asia, 2011.

[8] E. Lach, W. Idler, "Modulation formats for $100 \mathrm{G}$ and beyond", in Optical Fiber Technology, vol. 17, pp. 377-386, 2011. [Online]. Available: http://dx.doi.org/10.1016/j.yofte.2011.07.012

[9] E. Ip, A. Lau, D. Barros, J. Kahn, "Coherent detection in optical fiber systems”, Opt. Express, vol. 16, no. 2, pp. 753-791, 2008. [Online] Available: http://dx.doi.org/10.1364/OE.16.000753

[10] J. Renaudier, O. Bertran-Pardo, G. Charlet, M. Salsi, H. Mardoyan, P. Tran, S. Bigo, "8 Tb/s long haul transmission over low dispersion fibers using $100 \mathrm{~Gb} / \mathrm{s}$ PDM-QPSK channels paired with coherent detection", Bell Labs Technical Journal, vol. 14, no. 4, pp. 27-45, 2010. [Online]. Available: http://dx.doi.org/10.1002/bltj.20402 
[11] B. Zhu, D. W. Peckham, X. Jiang, R. Lingle Jr, "System performance of long-haul 112-Gb/s PDM-QPSK DWDM transmission over largearea fiber and SSMF spans", in 39th European Conf. and Exhibition Optical Communication (ECOC 2013), 2013. [Online]. Available: http://dx.doi.org/10.1049/cp.2013.1625

[12] J. Karaki, E. Pincemin, D. Grot, T.Guiliossou, Y. Jaouen, R. le Bidan T. le Gall, "Dual-polarization multi-band OFDM versus single-carrier DP-QPSK for 100 Gbps long-haul WDM transmission over legacy infrastructure", in 38th European Conf. and Exhibition Optical Communications (ECOC), 2012. [Online]. Available: http://dx.doi.org/10.1364/ECEOC.2012.P4.17

[13] T. J. Xia, G. A. Wellbrock, M. Huang, S. Zhang, Y. Huang, D. Chang, S. Burtsev, W. Pelouch, E. Zak, H. de Pedro, W. Szeto, H. Fevrier, "Transmission of 400G PM-16QAM channels over longhaul distance with commercial all-distributed raman amplification system and aged standard SMF in field", in Optical Fiber Communications Conf. and Exhibition (OFC), 2014. [Online]. Available: http://dx.doi.org/10.1364/OFC.2014.Tu2B.1

[14] X. Zhou, L. E. Nelson, P. Magill, R. Isaac, B. Zhu, D. W. Peckham, P. I. Borel, K. Carlson, "High spectral efficiency $400 \mathrm{~Gb} / \mathrm{s}$ transmission using PDM time-domain hybrid 32-64 QAM and training-assisted carrier recovery", Journal of Lightwave Technology, vol. 31, no. 7, pp. 999-1005, 2013. [Online]. Available: http://dx.doi.org/10.1109/JLT.2013.2243643

[15] J. D. Downie, J. Hurley, D. Pikula, "Transmission of $256 \mathrm{~Gb} / \mathrm{s} \mathrm{PM-}$ 16QAM and $128 \mathrm{~Gb} / \mathrm{s}$ PM-QPSK signals over long-haul and submarine systems with span lengths greater than $100 \mathrm{~km}$ ", in 39th European Conf. and Exhibition Optical Communication (ECOC 2013), 2013. [Online]. Available: http://dx.doi.org/10.1049/ cp.2013.1345

[16] W. Freude, R. Schmogrow, B. Nebendahl, M. Winter, A. Josten, D, Hillerkuss, S. Koenig, J. Meyer, M. Dreschmann, M. Huebner, C. Koos, J. Becker, J. Leuthold, "Quality metrics for optical signals: eye diagram, Q-factor, OSNR, EVM and BER", in Proc. 14th Int Conf. Transparent Optical Networks (ICTON), 2012, pp. 1-4.

[17] OptSim models reference, Volume I Sample mode, RSOFT Design Group, Inc., 2010.

[18] OptSim models reference, Volume I Block mode, RSOFT Design Group, Inc., 2010.

[19] Ch. Laperle, B. Villeneuve, Zh. Zhang, D. Mcghan, H. Sun, M. O'Sullivan, "WDM performance and PMD tolerance of a coherent 40-Gbit/s dual-polarization QPSK transceiver", Journal of Lightwave Technology, vol. 26, no. 1, 2008. [Online]. Available: http://dx.doi.org/10.1109/JLT. 2007.913071 